\title{
Role of hematopoietic stem cell transplantation in Philadelphia chromosome- positive acute lymphoblastic leukemia: a report of 2 unique cases
}

\author{
Yadanar Lwin'1 , David Ma ${ }^{1,2}$ \\ ${ }^{1}$ Department of Haematology and BM Transplant, St Vincent's Hospital Sydney, NSW, Australia \\ ${ }^{2}$ St Vincent's Clinical School, Faculty of Medicine, the University of New South Wales
}

Abstract

We report, to our knowledge, the first reported case of Philadelphia chromosome-positive acute lymphoblastic leukemia (Ph+ALL) developing 26 years after allogeneic stem cell transplantation for chronic myeloid leukemia $(\mathrm{CML})$. The second case involved de novo $\mathrm{Ph}+\mathrm{ALL}$ that developed 16 years post-autologous stem cell transplantation in sustained molecular remission. These cases illustrate and raise some key issues in the management of patients with $\mathrm{Ph}+$ leukemias, including the role of hematopoietic stem cell transplantation, tyrosine kinase inhibitors, and long-term post-transplantation follow-up.

Key words: acute lymphoblastic leukemia, tyrosine kinase inhibitor, chronic myeloid leukemia, hematopoietic stem cell transplantation.

Submitted March 25, 2019; Accepted April 5, 2019; Published online June 7, 2019; Issued online August 1, 2019

Correspondence: David DF Ma, Department of Haematology and BM Transplant, St Vincent's Hospital, Sydney 370 Victoria Street, Darlinghurst, NSW, 2010, Australia, E-mail: a.laferty@amr.org.au

\section{Introduction}

Philadelphia chromosome-positive acute lymphoblastic leukemia (ALL) ( Ph + ALL) is a biologically and clinically distinct type of ALL associated with poor prognosis ${ }^{1} . \mathrm{Ph}+\mathrm{ALL}$ can result from disease transformation or blast crisis of chronic myeloid leukemia (CML) . CML relapse more than 15 years after allogeneic (allo) hematopoietic stem cell transplantation (HSCT) is rare, and the development of $\mathrm{Ph}+\mathrm{ALL}$ following allo-HSCT for CML is also uncommon ${ }^{2}$. Our first case involved the development of $\mathrm{Ph}+$ ALL 26 years post-allo-HSCT for CML. Our second case was one of de novo Ph + ALL in molecular remission for 16 years after autologous (auto) HSCT, even after cessation of tyrosine kinase inhibitors (TKIs).

\section{Case Presentation}

\section{Case 1}

A 37-year-old man was originally diagnosed with chronic-phase CML following investigations for right eye retinal vein thrombosis in September 1990. After initial leukapheresis and hydroxycarbamide, he underwent allo-
HSCT from his 41-year-old human leukocyte antigen (HLA)-identical brother in August 1991. A myeloablative conditioning regimen comprised busulfan and cyclophosphamide, and he received graft-versus-host disease (GVHD) prophylaxis with methotrexate and cyclosporine. His post-transplant course was uneventful except for bacterial sepsis before engraftment, grade I acute GVHD and limited chronic GVHD of the skin. In August 2017, he was found to lose molecular response with a bcr-abl p210 transcript of 59\%. The patient was asymptomatic; blood counts were normal except for occasional circulating blasts. Bone marrow biopsy revealed a markedly hypercellular marrow with blasts of $90 \%$. The blasts were positive for cluster of differentiation (CD) 19, CD20, CD22, CD34, and HLA-DR. Cytogenetics showed 47,XY, t (9; 22) (q34; q11.2), + der (22) t (9;22) (q34; q11.2), and RT-PCR demonstrated BCR/ABL mRNA of b2a2 type. Cerebrospinal fluid analysis did not show central nervous system involvement. The patient received reinduction with GRAALL regimen ${ }^{3}$ and imatinib and achieved cytogenetic and molecular remission. In February 2018, he underwent second allo-HSCT with peripheral blood stem cells from his original sibling donor. He received reduced-intensity conditioning with 
fludarabine and melphalan, and GVHD prophylaxis consisted of methotrexate and cyclosporine. Imatinib was reinitiated on day 28 post-transplant. His post-transplant course was complicated by bacterial line sepsis and grade I acute skin GVHD. He achieved full donor chimerism post-transplant and remains in complete molecular remission to date.

\section{Case 2}

A 52-year-old man was diagnosed to have de novo $\mathrm{Ph}+\mathrm{ALL}$ in November 2001. He presented with a 6-week history of dyspnea, bruising, gum bleeding, and retroperitoneal bleeding. A full blood count revealed a white blood cell count of $118 \times 109 / \mathrm{L}$ with $91 \%$ blasts, a hemoglobin level of $117 \mathrm{~g} / \mathrm{L}$, and a platelet count of $9 \times$ $109 /$ L. Bone marrow biopsy showed a markedly hypercellular marrow with $96 \%$ blasts expressing CD10, CD19, CD20, CD22, CD34, and HLA-DR. Cytogenetics showed $\mathrm{t}(9 ; 22)$. He initially received imatinib, followed by multimodality chemotherapy (LALA-94 protocol) ${ }^{4}$. Morphologic and cytogenetic remission was achieved in February 2002, and he underwent auto-HSCT due to the unavailability of a suitable donor in May 2002. The conditioning regimen consisted of cyclophosphamide and busulfan. Apart from bacterial sepsis, his post-transplant course was uneventful; imatinib was recommenced 1 month post-transplant. He sustained cytogenetic remission post-transplant, and the bcr-abl transcript has remained undetectable since 2003. In September 2010, maintenance therapy containing imatinib was switched to dasatinib because of progressive sensory peripheral neuropathy, which was thought to be imatinib-related after comprehensive neurological assessment. The neuropathy subsequently resolved and remained quiescent for 6 years. In June 2016, dasatinib was discontinued after extensive investigations for progressive motor and sensory peripheral neuropathy. Subsequently, the symptoms improved, which was also supported by nerve conduction study findings. He is currently well and remains in complete molecular remission 17 years after diagnosis and 2 years after discontinuing TKIs.

\section{Discussion}

Although TKIs have replaced allo-HSCT as frontline therapy for CML, allo-HSCT remains an effective therapeutic option to produce durable remissions in patients resistant to TKIs and in those with advanced disease ${ }^{5}$. Disease phase at transplantation, absence of GVHD, and the use of T-cell-depleted grafts are the major risk factors of relapse ${ }^{6}$, but our first case lacked these factors. The majority of relapses after allo-HSCT for CML occur within the first 3 years, and so far, only one case of relapse with myeloid transformation (accelerated phase)
24 years after allografting has been reported ${ }^{2}$. Thus, our case highlights the risk of very late relapse of CML after allo-HSCT and a need for indefinite monitoring as effective salvage treatment is available.

An unusual feature of the second case was peripheral neuropathy, which developed in a clear temporal association with TKI use. Peripheral neuropathy is an extremely uncommon side effect of TKIs, and the underlying mechanism is unclear ${ }^{7,8}$. It may be due to direct toxic effects or off-target immune attacks on nerve myelin sheaths. One case report suggested drug interactions as a potential cause and recommended therapeutic monitoring of plasma imatinib levels ${ }^{7}$. In our case, no concomitant medication was administered.

The other uniqueness of our second case was the longterm disease-free survival after auto-HSCT, demonstrating auto-HSCT with TKIs as a suitable consolidation modality for Ph + ALL. Historically, Ph + ALL was considered a high-risk subtype and allo-HSCT was considered the standard of care for eligible patients despite significant treatment-related risks ${ }^{1}$. As the incorporation of TKIs in treatment regimens has resulted in improved clinical outcomes, frontline auto-HSCT plus TKIs has become a potential alternative option ${ }^{1,3,9}$. Long-term follow-up of de novo Ph + ALL patients in the GRAAPH2003 study reported a 4-year overall survival of up to $80 \%$ and 4 -year disease-free survival of $50 \%$ after auto$\mathrm{HSCT}^{3}$. A recent registry-based, retrospective analysis showed comparable outcomes of auto-HSCT and alloHSCT in patients with Ph + ALL in first molecular remission in the TKI era'.

In summary, both cases clearly emphasize the role of TKIs in the management of de novo or secondary $\mathrm{Ph}+$ ALL. However, there are no commonly accepted standards or strong evidence regarding the choice of TKI, dosage, time of initiation, or treatment duration ${ }^{10}$. Considering the associated adverse events, the effects on quality of life and economic impact of long-term TKI use, it is particularly important to investigate the possibility of safe treatment cessation. Further randomized control trials are warranted.

\section{Acknowledgements}

We thank Dr K Fay and Dr D Kliman for reviewing the manuscript and providing valuable suggestions.

\section{Author's contribution}

Design, manuscript writing and approval of manuscript: YL and DM. 


\section{Conflicts of Interest}

The authors declare no conflict of interest. Disclosure forms provided by the authors are available here.

\section{References}

1. El Fakih R, Kharfan-Dabaja MA, Aljurf M. Refining the Role of Hematopoietic Cell Transplantation for Acute Lymphoblastic Leukemia as Novel Therapies Emerge. Biol Blood Marrow Transplant 2016; 22: 2126-33.

2. Sekhri A, Liu D, Rasul M, Ahmed N, Ahmed T, Seiter K. Very late relapse of chronic myelogenous leukemia after allogeneic bone marrow transplantation. Leukemia research 2009; 33: 1291-3.

3. Tanguy-Schmidt A, Rousselot P, Chalandon Y, Cayuela JM, Hayette S, Vekemans MC, et al. Long-term follow-up of the imatinib GRAAPH-2003 study in newly diagnosed patients with de novo Philadelphia chromosome-positive acute lymphoblastic leukemia: a GRAALL study. Biol Blood Marrow Transplant 2013; 19: 150-5.

4. Thomas X, Boiron J-M, Huguet F, Dombret H, Bradstock K, Vey N, et al. Outcome of Treatment in Adults With Acute Lymphoblastic Leukemia: Analysis of the LALA-94 Trial. Journal of Clinical Oncology 2004; 22: 4075-86.

5. Jabbour E, Kantarjian H. Chronic myeloid leukemia: 2018 update on diagnosis, therapy and monitoring. American journal of hematology 2018; 93: 442-59.
6. Copelan EA, Crilley PA, Szer J, Dodds AJ, Stevenson D, Phillips G, et al. Late mortality and relapse following BuCy2 and HLA-identical sibling marrow transplantation for chronic myelogenous leukemia. Biol Blood Marrow Transplant 2009; 15: 851-5.

7. Ross DM. Peripheral neuropathy on imatinib treatment for chronic myeloid leukaemia: suspected adverse drug interaction with amlodipine. Intern Med J 2009; 39: 708.

8. Ishida T, Akagawa N, Miyata T, Tominaga N, Iizuka T, Higashihara $\mathrm{M}$, et al. Dasatinib-associated reversible demyelinating peripheral polyneuropathy in a case of chronic myeloid leukemia. International journal of hematology 2018; 107: 373-7.

9. Giebel S, Labopin M, Potter M, Poire X, Sengeloev H, Socie G, et al. Comparable results of autologous and allogeneic haematopoietic stem cell transplantation for adults with Philadelphiapositive acute lymphoblastic leukaemia in first complete molecular remission: An analysis by the Acute Leukemia Working Party of the EBMT. Eur J Cancer 2018; 96: 73-81.

10. Giebel S, Czyz A, Ottmann O, Baron F, Brissot E, Ciceri F, et al. Use of tyrosine kinase inhibitors to prevent relapse after allogeneic hematopoietic stem cell transplantation for patients with Philadelphia chromosome-positive acute lymphoblastic leukemia: A position statement of the Acute Leukemia Working Party of the European Society for Blood and Marrow Transplantation. Cancer 2016; 122: 2941-51.

https: //doi.org/10.31547/bct-2019-001

Copyright (C) 2019 APBMT. All Rights Reserved. 